\title{
RAA-Cas12a-Tg: A Nucleic Acid Detection System for Toxoplasma gondii Based on CRISPR-Cas12a Combined with Recombinase-Aided Amplification (RAA)
}

\author{
Qiao-Ni Ma ${ }^{1}$, Meng Wang ${ }^{1}$, Lai-Bao Zheng ${ }^{2} \oplus$, Zi-Qin Lin ${ }^{2}$, Muhammad Ehsan ${ }^{3} \oplus$, Xing-Xing Xiao ${ }^{2, *}$ \\ and Xing-Quan Zhu 1,4,5,*D \\ 1 State Key Laboratory of Veterinary Etiological Biology, Key Laboratory of Veterinary Parasitology \\ of Gansu Province, Lanzhou Veterinary Research Institute, Chinese Academy of Agricultural Sciences, \\ Lanzhou 730046, China; maxintan1228@gmail.com (Q.-N.M.); wangmeng02@caas.cn (M.W.) \\ 2 Wenzhou Key Laboratory of Sanitary Microbiology, Key Laboratory of Laboratory Medicine, \\ Ministry of Education, School of Laboratory Medicine and Life Sciences, Wenzhou Medical University, \\ Wenzhou 325035, China; zhenglaibao@wmu.edu.cn (L.-B.Z.); ziqinlin@wmu.edu.cn (Z.-Q.L.) \\ 3 Department of Parasitology, Faculty of Veterinary and Animal Sciences, \\ The Islamia University of Bahawalpur, Bahawalpur 63100, Pakistan; mehsan124@gmail.com \\ 4 College of Veterinary Medicine, Shanxi Agricultural University, Taigu, Jinzhong 030801, China \\ 5 Key Laboratory of Veterinary Public Health of Yunnan Province, College of Veterinary Medicine, \\ Yunnan Agricultural University, Kunming 650201, China \\ * Correspondence: xiaoxingxing@wmu.edu.cn (X.-X.X.); xingquanzhu1@hotmail.com (X.-Q.Z.)
}

\section{check for} updates

Citation: Ma, Q.-N.; Wang, M.; Zheng, L.-B.; Lin, Z.-Q.; Ehsan, M.; Xiao, X.-X.; Zhu, X.-Q. RAA-Cas12a-Tg: A Nucleic Acid Detection System for Toxoplasma gondii Based on CRISPR-Cas12a Combined with Recombinase-Aided Amplification (RAA). Microorganisms 2021, 9, 1644. https://doi.org/ 10.3390/microorganisms 9081644

Academic Editor: Ali Rostami

Received: 17 June 2021

Accepted: 22 July 2021

Published: 31 July 2021

Publisher's Note: MDPI stays neutral with regard to jurisdictional claims in published maps and institutional affiliations.

Copyright: (C) 2021 by the authors. Licensee MDPI, Basel, Switzerland. This article is an open access article distributed under the terms and conditions of the Creative Commons Attribution (CC BY) license (https:// creativecommons.org/licenses/by/ $4.0 /)$.

\begin{abstract}
Toxoplasmosis, caused by the intracellular protozoon Toxoplasma gondii, is a significant parasitic zoonosis with a world-wide distribution. As a main transmission route, human infection can be acquired by the ingestion of T. gondii oocysts from the environment (e.g., soil, water, fruits and vegetables). Regarding the detection of $T$. gondii oocysts in environmental samples, the development of a time-saving, cost-effective and highly sensitive technique is crucial for the surveillance, prevention and control of toxoplasmosis. In this study, we developed a new method by combining recombinase-aided amplification (RAA) with CRISPR-Cas12a, designated as the RAA-Cas12a-Tg system. Here, we compared this system targeting the $529 \mathrm{bp}$ repeat element ( $529 \mathrm{bp}-\mathrm{RE}$ ) with the routine PCR targeting both 529 bp-RE and ITS-1 gene, respectively, to assess its ability to detect T. gondii oocysts in soil samples. Our results indicated that the 529 bp RE-based RAA-Cas12a-Tg system was able to detect $T$. gondii successfully in nearly an hour at body temperature and was more sensitive than the routine PCR assay. The sensitivity of this system reached as low as $1 \mathrm{fM}$ with high specificity. Thus, RAA-Cas12a-Tg system provided a rapid, sensitive and easily operable method for point-of-care detection of T. gondii oocysts in soil, which will facilitate the control of T. gondii infection in humans and animals.
\end{abstract}

Keywords: Toxoplasma gondii; detection; RAA-Cas12a-Tg system; soil

\section{Introduction}

Toxoplasmosis is an important zoonotic disease caused by the protozoon Toxoplasma gondii, which is an opportunistic pathogen with the capacity of infecting nearly all warm-blooded animal species and one third of the world's human populations [1,2]. For example, approximately $10 \%$ of Chinese people were infected with T. gondii [2]. Without effective treatment and vaccines, infection with $T$. gondii can cause serious clinical symptoms and even fatal consequences in immunocompromised patients (e.g., HIV / ADIS and transplant patients) and congenitally infected infants [3,4]. Toxoplasmosis also can result in the abortion and stillbirth of livestock, causing considerable economic losses to animal husbandry [1,5]. As a foodborne and waterborne pathogen, T. gondii is capable of inadvertently appearing in vegetables, fruits, water and soil [6-13]. Human and animal infections occur through eating raw or undercooked meat 
or via the ingestion of water or food contaminated with sporulated oocysts [14]. Therefore, the rapid, highly specific and accurate detection of $T$. gondii from natural environment is crucial for the surveillance, prevention and control of toxoplasmosis.

Conventional diagnostic methods such as light microscope examination are timeconsuming and unreliable, and require a large volume of biological and environmental samples [15]. Serological diagnosis also has some limitations, for example, it is difficult to diagnose toxoplasmosis in immunocompromised patients [16]. However, molecular methods are independent of the host's immune response, and allow direct detection of the parasite [17]. Currently, molecular methods supporting the T. gondii detection are mainly emerging from PCR-based technologies, such as routine PCR, real-time PCR (RT-PCR), and loop-mediated isothermal amplification (LAMP) [15]. For these molecular technologies, T. gondii-specific repetitive DNA sequences, such as B1 gene, ITS-1 and 18S rDNA, and 529-bp repeat element (RE), have shown good sensitivities for the detection of T. gondii [15]. In contrast, PCR and LAMP assays targeting the 529-bp RE sequence have suggested that they are more sensitive than the B1 gene-based assays $[9,18,19]$, while the multicopy ITS-1 and 18S rDNA have shown a similar sensitivity of the B1 gene [20,21]. In addition to sensitivity evaluation of target molecules, LAMP is more sensitive than the routine PCR, but slightly lower than the RT-PCR [22,23]. Although RT-PCR-based methods now hold well performance especially for detecting low concentration of target DNA, the commercial RT-PCR assay is expensive, instrument-dependent and time-consuming. Therefore, the development of an easy, sensitive and cost-effective molecular method is warranted.

As it has the ability named trans-cleavage to accurately recognize and cleave specific nucleic acid target, CRISPR-Cas systems have shown great potential as practical diagnostic tools [24,25]. The Cas nucleases contain RNA-guided RNases (Cas13a and Cas13b) and RNA-guided DNases (Cas12a, Cas12b and Cas14), which are able to exhibit non-specific trans-cleavage activity after binding to their specific targets and has been fully evaluated for the detection of nucleic acids [26-28]. For Cas orthologues, Cas12a and Cas13 are the main nucleases for the development of CRISPR-Cas-based nucleic acid detection method with higher sensitivity and specificity for the capability of recognizing a nucleic acid target, and they also have been developed to combine with isothermal amplification technologies, such as Recombinase-aided Amplification (RAA), to enhance the diagnostic accuracy [29-31]. Depending on the type of nucleic acid substrate (DNA or RNA), CRISPR-Cas12a/Cas13based systems have been alternatively applied to the detection of different pathogens, including viruses [26,29,32-34], bacteria [31,35-37], parasitic protozoa Plasmodium spp. [38] and Cryptosporidium spp. [39]. However, to date, there has been no report of the use of CRISPR-Cas system for the detection of T. gondii yet.

In this work, by designing specific CRISPR-derived RNA (crRNA) probe targeting the $529 \mathrm{bp}$-RE sequence of $T$. gondii and combining RAA and Cas12a protein, termed RAA-Cas12a-Tg system, we presented an advanced approach for easy, rapid and accurate detection of T. gondii DNA by observing the fluorescence intensity with the naked eye under an ultraviolet light or via the detection of the fluorescence wavelength using a microplate reader (Figure 1). Additionally, by examination of 30 soil DNA samples from our previous study [9], we also compared the T. gondii-positive results detected by the conventional PCR-based method and RAA-Cas12a-Tg system. Our verification indicated that RAA-Cas12a-Tg system has merits in the aspect of conveniency and sensitivity, and its applications may facilitate the control of toxoplasmosis in humans and animals. 


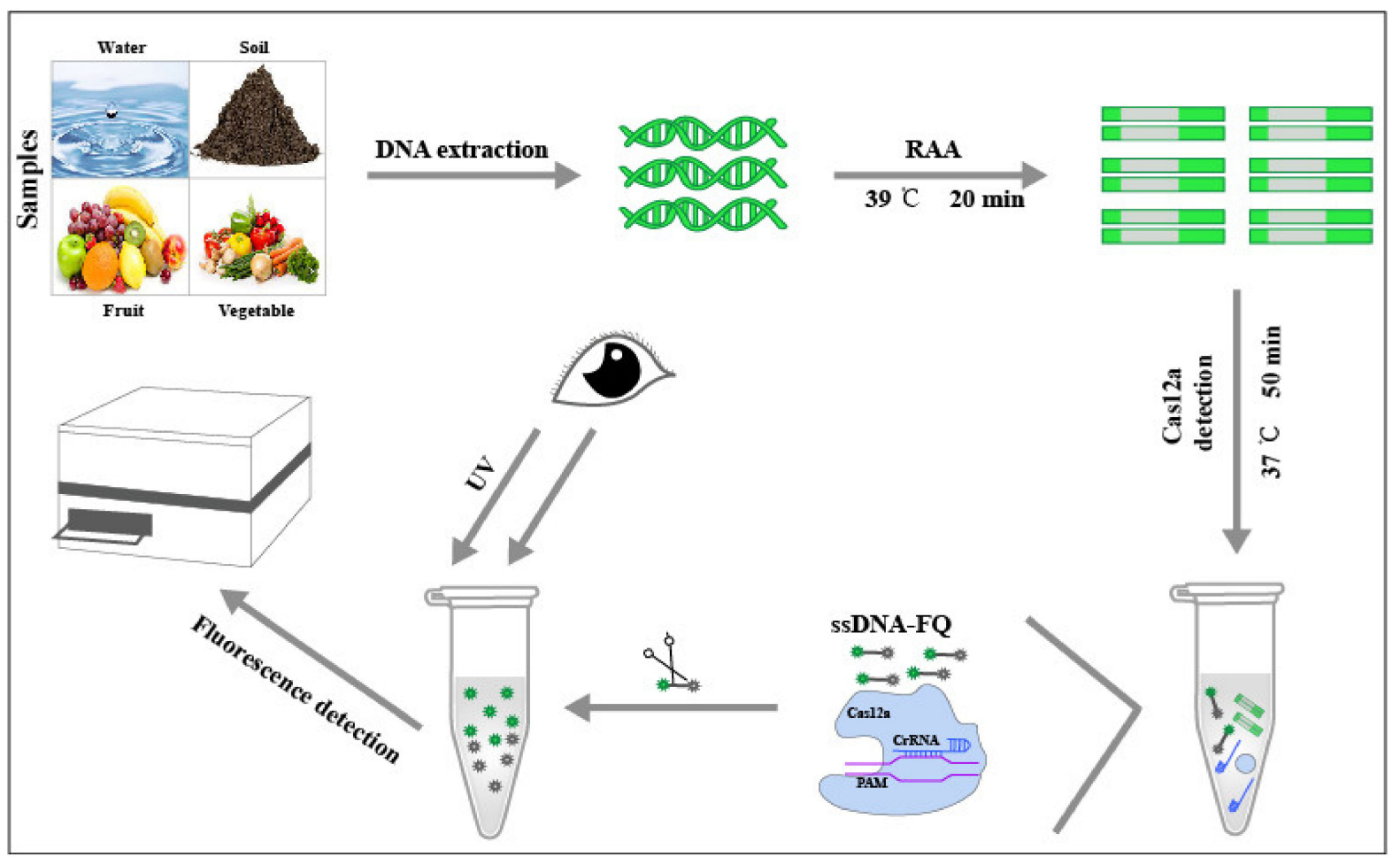

Figure 1. Schematic diagram of the application of the RAA-Cas12a-Tg detection system to environmental samples such as soil, water, fruit and vegetable.

2. Materials and Methods

2.1. Materials

All primers used for RAA assay and PCR amplifications were synthesized by TSINGKE Biotech (Xi'an, China). ssDNA-FQ was synthesized by Sangon Biotech (Shanghai, China) and crRNA was synthesized by GenePharma (Shanghai, China). The basic RAA kits were purchased from ZC Bio-Sci\&Tech (Hangzhou, China). A total of 30 soil DNA samples were provided by Dr. Wei Cong of Marine College, Shandong University, China [9]. Seven parasite DNA samples (T. gondii, Cryptosporidium parvum, Neospora caninum, Enterocytozoon bieneusi, Blastocystis sp., Eimeria tenella, and Toxocara canis) were prepared by our lab and stored at $-20^{\circ} \mathrm{C}$ until for RAA- and PCR-based assays.

\subsection{Establishment of 529 bp RE-Based RAA Assay}

Four pairs of RAA primers were designed using Primer Premier 5 software [40]. The primer sequences and lengths are summarized in Table 1. The nucleotide sequence of T. gondii 529 bp-RE (RH strain) was downloaded from NCBI GenBank (https:/ / www. ncbi.nlm.nih.gov/genbank/, accessed on 20 November 2020) with accession number AF146527.1 [18]. The obtained DNA of each sample was used for RAA reaction using RAA kit assay. Using $2 \mu \mathrm{L}$ of DNA template, a total $50 \mu \mathrm{L}$ reaction system was performed in a $200 \mu \mathrm{L}$ aseptic single tube that contained lyophilized powder, with $41.5 \mu \mathrm{L}$ of buffer A, $2 \mu \mathrm{L}$ each of forward and reverse primers and $2.5 \mu \mathrm{L}$ of buffer B. The reaction tube was inverted 6 8 times and then centrifuged for $15 \mathrm{~s}$. The reaction tube was placed on a Thermostatic Water Bath, and then incubated at $39{ }^{\circ} \mathrm{C}$ for $20 \mathrm{~min}$. The amplification products were purified using Universal DNA Purification Kit (Tiangen, Beijing, China). 
Table 1. Primer sequences for RAA, plasmid, crRNA and ssDNA-FQ.

\begin{tabular}{|c|c|c|c|}
\hline Assay & Primer Name & Sequence $\left(5^{\prime} \rightarrow 3^{\prime}\right)$ & Product Size $(b p)$ \\
\hline \multirow[t]{8}{*}{ RAA } & 529bp-RAA-F1 & GAAGGGACAGAAGTCGAAGGGGA & \multirow{2}{*}{171} \\
\hline & 529bp-RAA-R1 & GAAAAGCAGCCAAGCCGGAAACA & \\
\hline & 529bp-RAA-F2 & TGGAGCCACAGAAGGGACAGAAGT & \multirow[b]{2}{*}{184} \\
\hline & $529 \mathrm{bp}-\mathrm{RAA}-\mathrm{R} 2$ & CAGGAAAAGCAGCCAAGCCGGAAA & \\
\hline & 529bp-RAA-F3 & CAGAAGGGACAGAAGTCGAAGGGGA & \multirow[b]{2}{*}{175} \\
\hline & 529bp-RAA-R3 & AGGAAAAGCAGCCAAGCCGGAAACA & \\
\hline & $529 b p-R A A-F 4$ & GAGCCACAGAAGGGACAGAAGTCG & \multirow{2}{*}{186} \\
\hline & 529bp-RAA-R4 & CCTCCAGGAAAAGCAGCCAAGCCG & \\
\hline \multirow[t]{2}{*}{ Plasmid } & $529 \mathrm{bp}-\mathrm{PF}$ & GGAGGAAGACGAAAGTTG & \multirow{7}{*}{515} \\
\hline & $529 \mathrm{bp}-\mathrm{PR}$ & ACAGTGCATCTGGATTCC & \\
\hline \multirow[t]{4}{*}{ crRNA } & crRNA1 & UAAUUUCUACUAAGUGUAGAUACTCGGGCCCAGCTGCGTCT & \\
\hline & crRNA2 & UAAUUUCUACUAAGUGUAGAUACAGGCAAGCTCGCCTGTGC & \\
\hline & crRNA3 & UAAUUUCUACUAAGUGUAGAUCACCCUCCAGGAAAAGCAGCCA & \\
\hline & crRNA4 & UAAUUUCUACUAAGUGUAGAUCTCGTGGTGATGGCGGAGAG & \\
\hline ssDNA-FQ & $\operatorname{TgCas} 12 \mathrm{a}$ & 6FAM-CCGGAAAAAAAAAAAACCGG-BHQ1 & \\
\hline
\end{tabular}

\subsection{Construction of Positive Recombinant Plasmids pMD18-T-529 bp}

As shown in Table 1, we first designed a pair of plasmid primer based on the $529 \mathrm{bp}-\mathrm{RE}$ sequence using Primer Premier 5 software in order to establish the RAA-Cas12a-Tg system and to evaluate the sensitivity of this method. The $25 \mu \mathrm{L}$ PCR reaction system contained 12.5 $\mu \mathrm{L}$ Pre-Mix Taq Enzyme, $1.5 \mu \mathrm{L}$ each of forward and reverse primers, $2 \mu \mathrm{L}$ DNA template, and $7.5 \mu \mathrm{L}$ dd $\mathrm{H}_{2} \mathrm{O}$. The PCR was performed under the following cycling conditions: initial denaturation at $94{ }^{\circ} \mathrm{C}$ for $5 \mathrm{~min}$, followed by 34 cycles of $94{ }^{\circ} \mathrm{C}$ for $30 \mathrm{~s}, 57{ }^{\circ} \mathrm{C}$ for $30 \mathrm{~s}$ and $72{ }^{\circ} \mathrm{C}$ for $1 \mathrm{~min}$, with an additional $5 \mathrm{~min}$ final extension at $72{ }^{\circ} \mathrm{C}$. PCR products were examined by $1 \%$ agarose gel electrophoresis and then were recovered using E.Z.N.A ${ }^{\mathrm{TM}}$ Plasmid Mini Kit (OMEGA Bio-Tek, Norcross, GA, USA). Then, the gel recovered products were cloned into the $\mathrm{pMD}^{\mathrm{TM}} 18-\mathrm{T}$ vector (Takara, Shanghai, China). Finally, the concentration of positive recombinant plasmids was qualified using with the NanoPhotometer (IMPLEN, Munich, Germany), and the recombinant plasmid concentration was diluted from $10^{2}$ to $10^{-7} \mathrm{nM}$, and then stored at $-20{ }^{\circ} \mathrm{C}$ for the subsequent fluorescent detection of the Cas12a assay.

\section{4. crRNA/ssDNA-FQ Preparation and Fluorescence Detection of Cas12a}

The CRISPR-derived RNA (crRNA) and ssDNA-FQ sequences, as shown in Table 1, were designed based on Cas12a-mediated fluorescence detection reported by Zhang et al. [41]. crRNA, ssDNA-FQ reporter, EnGen Lba Cas12a protein (NEB, Ipswich, MA, USA), 10x NEBuffer 2.0 (NEB, Ipswich, MA, USA) and positive recombinant plasmids pMD ${ }^{\mathrm{TM}} 18-\mathrm{T}$ 529-bp (Takara, Shanghai, China) were used for Cas12a-mediated fluorescence detection. The processes of fluorescence detection was performed under the following procedures: (i) $800 \mathrm{nM}$ Cas12a and $1 \mu \mathrm{M}$ crRNA each of $20 \mu \mathrm{L}$ were simultaneously added to a $200 \mu \mathrm{L}$ aseptic PCR tube, and then incubated at $37^{\circ} \mathrm{C}$ for $20 \mathrm{~min}$; (ii) recombinant plasmids and $1 \mu \mathrm{M}$ ssDNA-FQ reporter each of $20 \mu \mathrm{L}$ were simultaneously added to the PCR tube of first-step, and then incubated at $37^{\circ} \mathrm{C}$ for $30 \mathrm{~min}$; (iii) after the aforementioned reaction, the final products were transferred into a black 96-well plate to detect the fluorescence with an excitation wavelength of $490 \mathrm{~nm}$, and the fluorescence was detected at wavelength of $510 \mathrm{~nm}$ to $600 \mathrm{~nm}$ using the Varioskan ${ }^{\mathrm{TM}}$ LUX microplate reader (Thermo Scientific, Waltham, MA, USA). During all the procedures, the concentration of crRNA, ssDNA-FQ reporter, Cas12a Protein and recombinant plasmids were diluted using 1x NEBuffer 2.0 (NEB, Ipswich, MA, USA) according to the actual requirements.

\subsection{Analysis of Specificity and Sensitivity of RAA-Cas12a System}

Each of $2 \mu \mathrm{L}$ DNA templates from T. gondii and the six "control" parasites were used for RAA reaction, respectively, and then we tested the specificity of RAA-Cas2a-Tg 
system by comparing T. gondii targeting the $529 \mathrm{bp}$-RE with other parasite DNA templates targeting the $529 \mathrm{bp}$-RE by Cas12a-mediated fluorescence detection. In terms of sensitivity detection experiments, 10 -fold serial dilutions ranging from $10^{2}$ to $10^{-7} \mathrm{nM}$ of the positive recombinant plasmids were utilized, and by mean of Student's t-test we also compared the detection sensitivity differences between different diluted plasmid DNA concentrations.

\subsection{Application of 529 bp RE-Based RAA-Cas12a System Detection to Soil Samples}

The performance of 529 bp RE-based RAA-Cas12a-Tg system was evaluated using 30 environmental soil DNA samples. Briefly, $2 \mu \mathrm{L}$ of soil DNA samples were used for RAA reaction, and the procedure was completed in $20 \mathrm{~min} ; 20 \mu \mathrm{L}$ of RAA amplification product was added to the Cas12a-mediated fluorescence system and the fluorescence detection was completed in $50 \mathrm{~min}$. We compared the detected results between RAA-Cas12a-Tg system and conventional PCR-based detection, and positive PCR products were sent to TSINGKE Biotech (Xi'an, China) for DNA sequencing.

\section{Results}

\subsection{Optimization of the RAA System}

Four pairs of 529 bp RE-based RAA primers (F1/R1, F2/R2, F3/R3 and F4/R4) were designed and, as shown in Table 1 , these primers were identified by RAA amplification, respectively. By comparison, the agarose gel electrophoresis (AGE) results showed that the $\mathrm{P}(\mathrm{F} 4+\mathrm{R} 4)$ primer combination had a relatively specific band and high intensity at 186 $\mathrm{bp}$, and the negative group $\mathrm{N}(\mathrm{F} 4+\mathrm{R} 4)$ did not present any band (Figure 2A). Thus, the $\mathrm{P}(\mathrm{F} 4+\mathrm{R} 4)$ primer combination was considered as the optimal primer pair for RAA system optimization. With regard to the RAA reaction temperature, five gradients including 25 , $30,39,42$, and $45^{\circ} \mathrm{C}$ were chosen for RAA reaction, and all of them were able to amplify a clear band with $186 \mathrm{bp}$ size in the AGE results (Figure $2 \mathrm{~B}$ ). In addition, we chose $39^{\circ} \mathrm{C}$ as the optimal temperature to determine the RAA reaction time and found that the DNA targeting 529 bp-RE could result in a clear band at $186 \mathrm{bp}$ at five different time points (i.e., 5, 10, 20, 30 and $40 \mathrm{~min}$ ) (Figure 2C). Thus, RAA reaction at $20 \mathrm{~min}$ was used for further experiment.

\subsection{Optimization of Cas12a-Mediated Fluorescence Detection Assay}

Four crRNA sequences (crRNA1, crRNA2, crRNA3 and crRNA4) were designed and prepared (Table 1), and we used the Cas12a-mediated fluorescence system to detect the crRNA-guided Cas12a cleavage activity and to select a suitable crRNA for further experiment. Within the emission wavelength of 510 to $600 \mathrm{~nm}$, as shown in Figure 3A, all four crRNAs and the crRNA-Mix (mixed in equal proportions for these crRNA) had a fluorescence intensity and reached the highest fluorescence value (plateau phase) at $520 \mathrm{~nm}$. From this result, we found that crRNA3 illustrated a better curve performance within the whole emission wavelength, and therefore this crRNA was considered as the best one for the Cas12a-mediated fluorescence detection assay. Additionally, we optimized the concentration of Cas12a protein in our Cas12a-mediated fluorescence detection assay, as the Cas12a concentration plays a crucial role in the detection of fluorescence. Four different concentration gradient values of Cas12a protein, including 200, 500, 800 and $1000 \mathrm{nM}$, were tested in this study. As shown in Figure 3B, the results showed that all concentration values had fluorescence signal and, relatively, the concentration at $800 \mathrm{nM}$ had much higher fluorescence intensity than that of other three concentration values (i.e., 200, 500 and $1000 \mathrm{nM}$ ) within the emission wavelength of 510 to $600 \mathrm{~nm}$. Thus, the concentration at $800 \mathrm{nM}$ was chosen as the optimal reaction value for Case12a protein. 

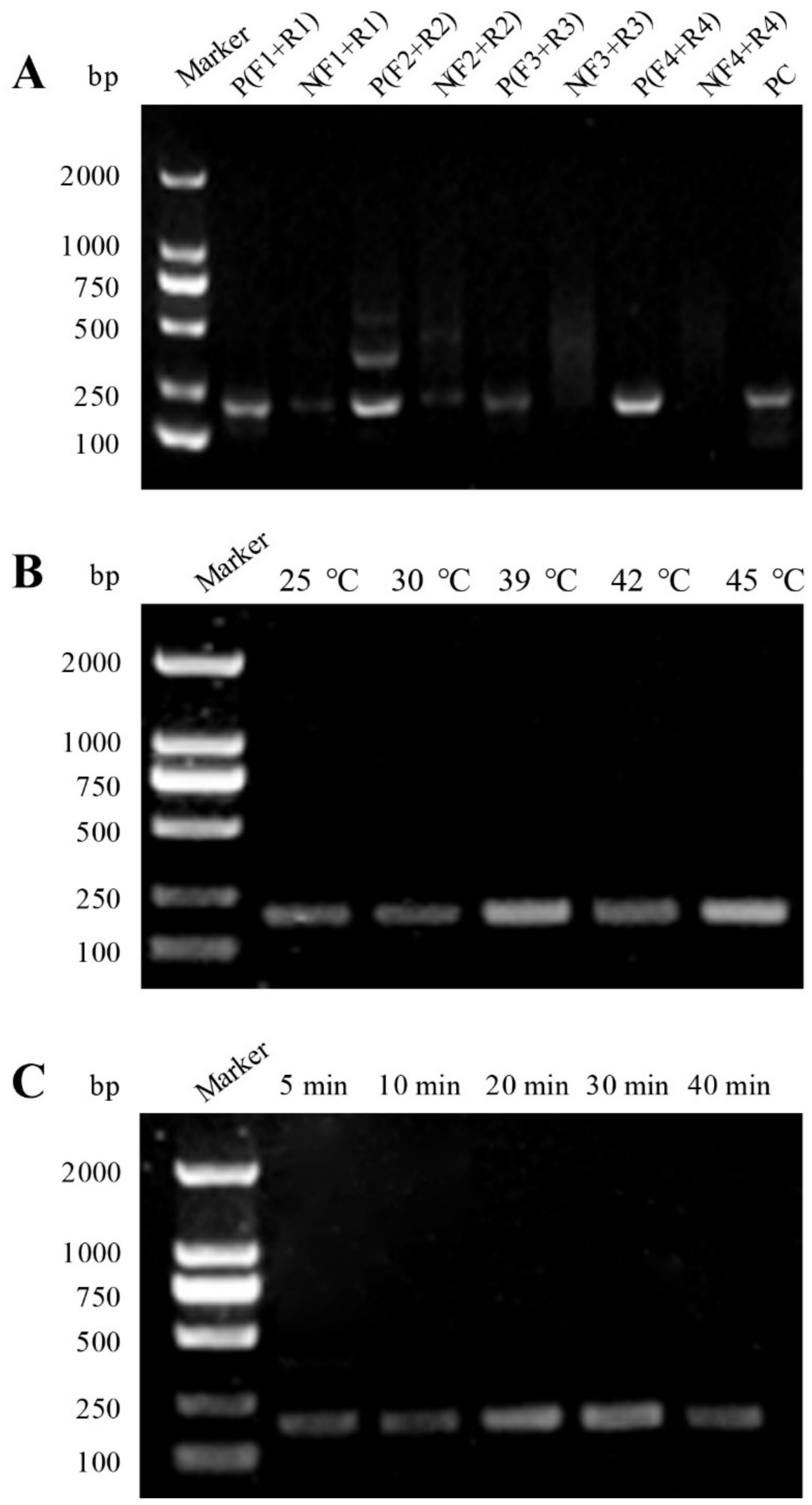

Figure 2. Optimization of the RAA assay. (A) Screening of the RAA primers. Agarose gel electrophoresis of RAA amplification products using different primer combinations. (B) Optimization of reaction temperature of the RAA assay. (C) Optimization of reaction time of the RAA assay. 

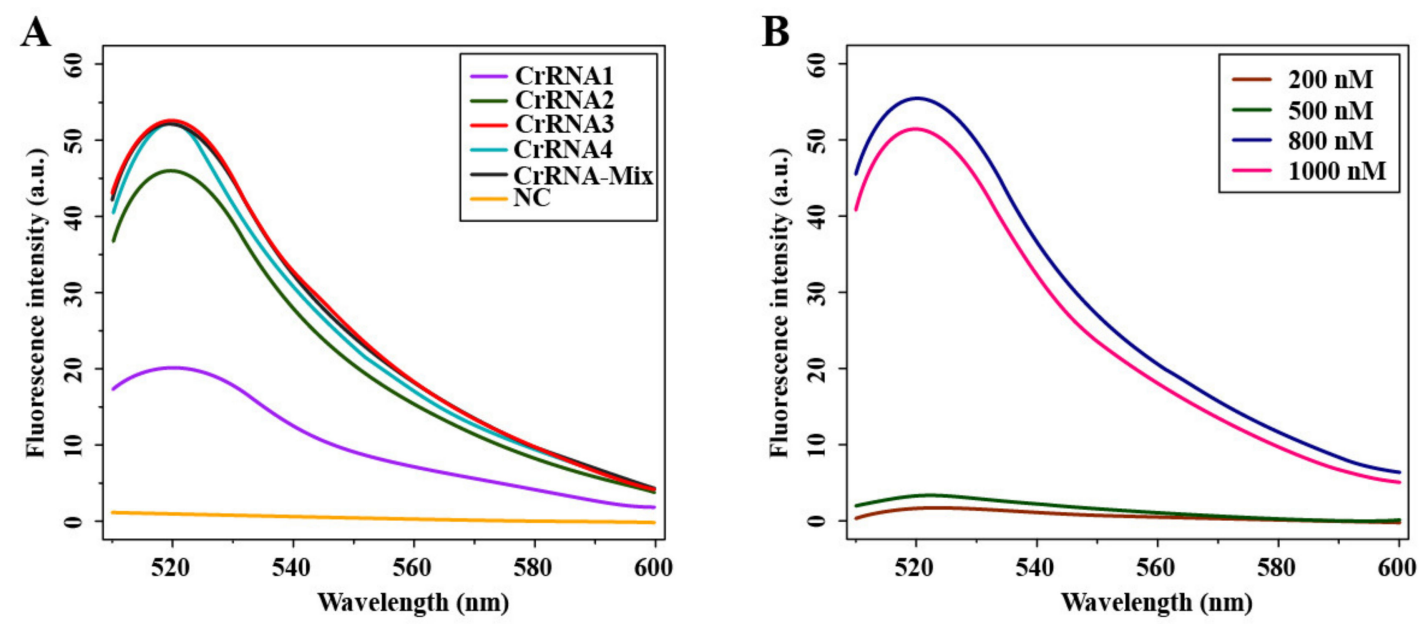

Figure 3. Optimization of the fluorescence detection of Cas12a. (A) Screening of the crRNA sequence. crRNA1, crRNA2, crRNA3 and crRNA4 represent different crRNAs; crRNA-MIX represents the product of the above four crRNAs mixed in the same proportion; NC stands for negative control. (B) Optimization of the concentration of LbCas12a.

\subsection{Evaluation of Sensitivity and Specificity of the RAA-Cas12a-Tg System}

To evaluate the sensitivity of the RAA-Cas12a system, the positive recombinant pMD18-T-529 bp plasmids diluted by 10-fold serial (ranging from $10^{2}$ to $10^{-7} \mathrm{nM}$ ) were used for fluorescence intensity test at excitation wavelength of $510 \mathrm{~nm}$ to $600 \mathrm{~nm}$. The statistical analysis revealed that the plasmids ranging from $10^{2}$ to $10^{-6} \mathrm{nM}$ had significantly higher fluorescence intensity than that of the negative control group ${ }^{* * * *} p<0.0001$ and $\left.{ }^{* * *} p<0.001\right)$; however, there was no significant difference between the group with $10^{-7} \mathrm{nM}$ and NC group (ns $p>0.05$ ) (Figure 4A). Visual detection of signal amplification demonstrated that the positive sample containing T. gondii DNA had an obvious signal by the naked eye under a UV transilluminator and bear a maximum test-band fluorescence intensity at the plateau phase $(520 \mathrm{~nm})$ by observation using a microplate reader, while the negative sample without $T$. gondii DNA did not exhibit any signal amplification using these two methods (Figure 4B).

We also evaluated the specificity by experimentally detecting and comparing the fluorescence intensity for T. gondii and other selected control parasites (C. parvum, N. caninum, E. bieneusi, Blastocystis sp., E. tenella and T. canis). As shown in Figure 4C, the results showed that fluorescence signal for $T$. gondii detection was significantly higher than that of other parasites and the NC group, and the largest fluorescence intensity fold between $T$. gondii and other parasites (average value) at plateau phase was able to reach 40 -fold. Additionally, analysis of relative fluorescence intensity showed that RAA-Cas12a-Tg system for T. gondii detection was more significant than that of the genetically closely and distant parasites $\left({ }^{* * * *} p<0.0001\right)$ (Figure 4D). These results suggested that the RAA-Cas12a system for T. gondii detection had sufficient specificity.

\subsection{Application of the 529 bp RE-Based RAA-Cas12a-Tg System for T. gondii Detection in Soil Samples}

We examined 30 soil DNA samples using the RAA-Cas12a-Tg system, and samples with relative fluorescence intensity were further evaluated by DNA sequencing. The results showed that 12 out of the 30 soil DNA samples were T. gondii positive detected by the RAACas12a-Tg system (Figure 5A) and samples with relative fluorescence intensity $>9$ were considered positive according to the result of sequencing (Figure 5B), while 10 samples were positive for T. gondii by conventional PCR method targeting both the 529-RE sequence and the ITS-1 rDNA [9], which was $100 \%$ consistent with 10 out of the 12 positive samples by our RAA-Cas12a-Tg system. Meanwhile, all positive samples were sequenced by TSINGKE Biotech (Xi'an, China), and the results showed that all the sequences represented the $529 \mathrm{bp}$ 
repeat element of $T$. gondii. Thus, the RAA-Cas12a-Tg system were more sensitive than the conventional PCR method.
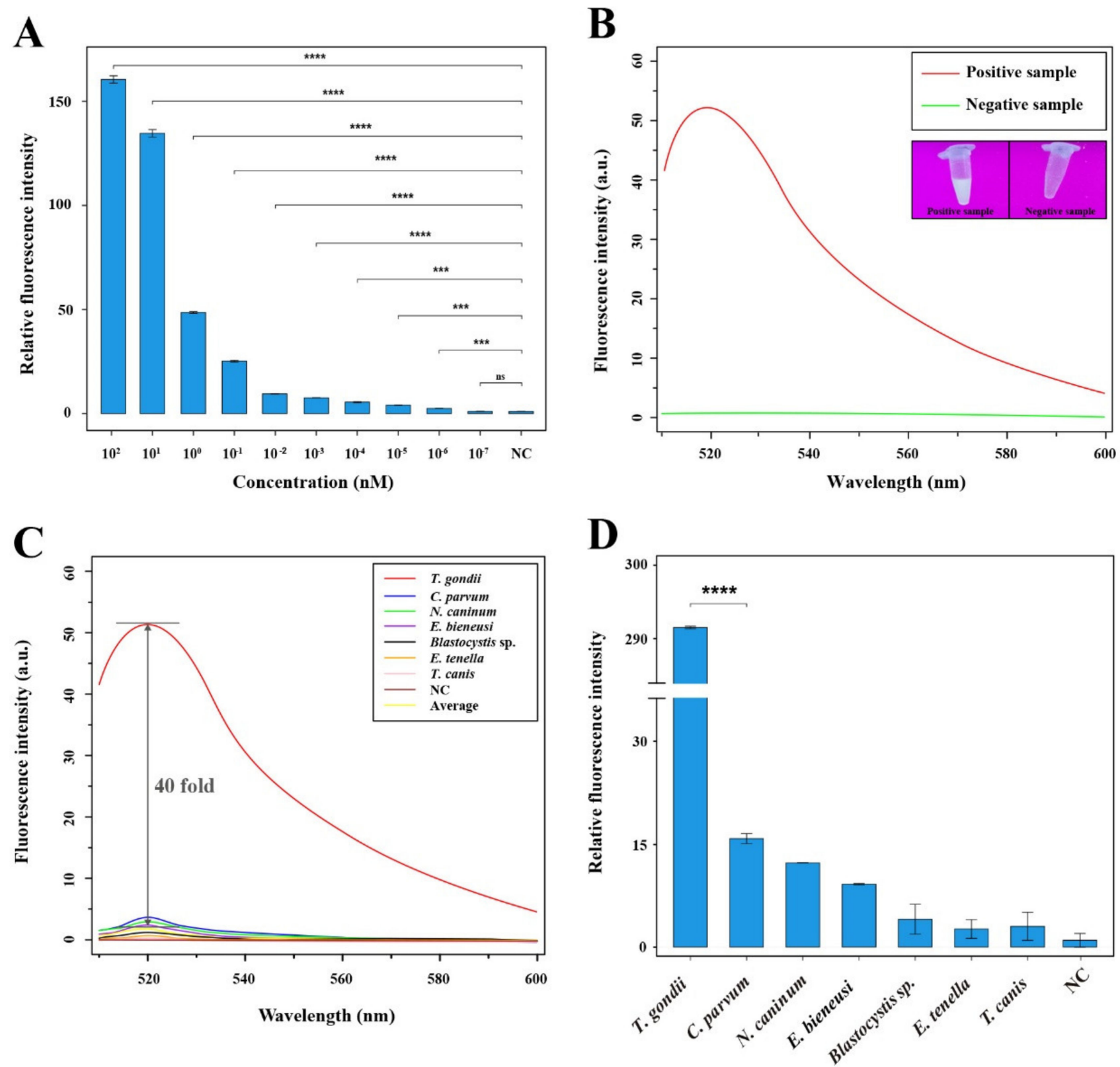

Figure 4. Analysis of specificity and sensitivity of the RAA-Cas12a-Tg system. (A) Sensitivity of the RAA-Cas12a system for T. gondii detection. Relative fluorescence intensity was estimated by the formula of (Ft-F0)/(Fn-F0) $\times 100 \%$, where Ft, F0, and Fn represent the fluorescence peak values of the positive recombinant pMD18-T-529 bp plasmids after dilution by 10-fold serial ranging from $10^{2}$ to $10^{-7} \mathrm{nM}$, blank, and negative control, respectively. Error bars represent the mean standard deviation (SD), where $n=2$ replicates. ${ }^{* * *} p \leq 0.0001 ;{ }^{* * *} p \leq 0.001 ;$ ns $p>0.05$. (B) Visual detection of signal amplification by the naked eye under a UV transilluminator and by an observation of microplate reader of the positive sample containing T. gondii DNA and the negative sample without T. gondii DNA. (C) Specificity of the RAA-Cas12a system for T. gondii detection. The nucleic acids of other six parasites including C. parvum, N. caninum, E. bieneusi, Blastocystis sp., E. tenella and T. canis were used to evaluate the specificity of the RAA-Cas12a-Tg system. Average represents the largest fluorescence intensity of other six parasites at plateau phase; NC stands for negative control. (D) Specificity of the RAA-Cas12a system for T. gondii detection. The relative fluorescence intensity of T. gondii detection using the RAA-Cas12a-Tg system was more significant than that of the closely related C. parvum and other five parasites. Error bars represent the mean SD, where $n=2$ replicates. ${ }^{* * * *} p \leq 0.0001$. 

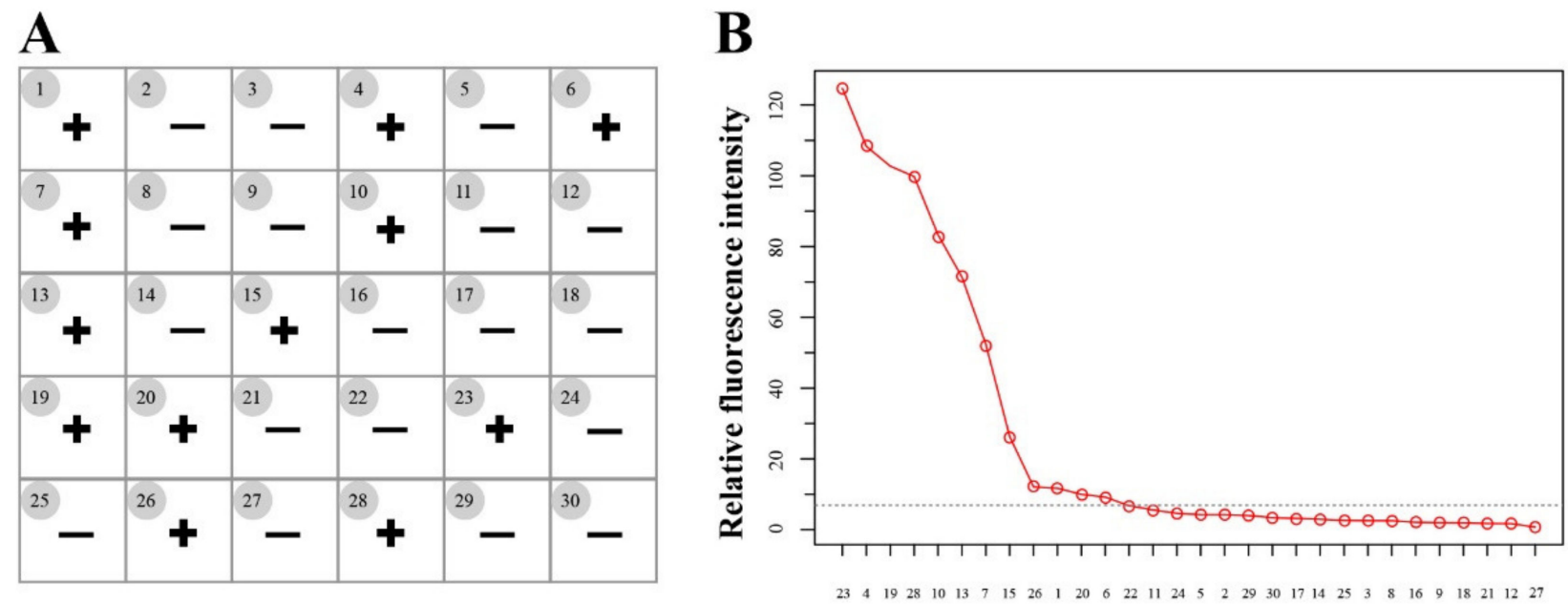

Sample code

Figure 5. Application of the RAA-Cas12a-Tg system to T. gondii detection in the environment samples. (A) Summary of T. gondii detection in soil samples $(n=30)$ using the RAA-Cas12a-Tg system. + means positive and - means negative. (B) 30 soil samples with relative fluorescence intensity $>9$ were considered as positive.

\section{Discussion}

Rapid and accurate detection of potential T. gondii DNA in the environment plays an important role in identifying, assessing and managing health and safety risks caused by the parasite, especially for emerging public health problems linked to food-, water- and soilborne outbreaks $[9,12,42-45]$. In the present study, we established a technique, designated as RAA-Cas12a-Tg system, for the detection of T. gondii DNA in soil. This system does not require an incubator higher than $39^{\circ} \mathrm{C}$, and its reaction can even be completed at the temperature of $\sim 37^{\circ} \mathrm{C}$. The whole reaction comprises test reagents that can be easily stored in a low-temperature vessel, and without the need of sophisticated technical requirements (Figure 1). The whole reaction process used in the study takes nearly $1 \mathrm{~h}$, and the results for highly copied nucleic acid can be easily obtained by ultraviolet irradiation with the naked eye (Figure 4B), or a handheld microplate reader device [46]. Therefore, the RAA-Cas12a$\mathrm{Tg}$ system is particularly suitable for an on-site T. gondii detection (e.g., sewage plant, park or farm) in a short time and without the requirement of an available diagnostic laboratory.

The option of an appropriate nucleic acid-amplified technology to combine with the CRISPR-Ca12a system plays a critical role in the clinical diagnosis. The common PCRbased technologies such as routine PCR and RT-PCR amplifications require a thermal cycle (usually 95-60-72 ${ }^{\circ} \mathrm{C}$ ) for more than $2 \mathrm{~h}$, while LAMP amplification requires a constant temperature of $\sim 65^{\circ} \mathrm{C}$ for more than $1 \mathrm{~h}$, and the primer design of LAMP assay is cumbersome. Thus, PCR, RT-PCR and LAMP technologies combining a CRISPR-Ca12a system are bulky and not suitable for rapid diagnosis. Furthermore, the Cas12a assay needs to be carried out at a temperature below $45^{\circ} \mathrm{C}$, otherwise the Cas12a nuclease would appear irreversible damage thereby affecting the cleavage rate of the CRISPR system [30,47]. Hence, if a singletube one-step reaction is performed, PCR, RT-PCR and LAMP technologies are also not suitable for combination with a CRISPR-Ca12a system. In the present study, the RAA system is a good option as the initial signal amplification, because RAA assay is much cheaper, just takes $20 \mathrm{~min}$ or lesser time, and the required temperature $\left(37 \sim 45^{\circ} \mathrm{C}\right)$ is consistent with the optional temperature of CRISPR-Ca12a system. Additionally, we also optimized the RAA system by testing primers, reaction temperatures and times (Figure $2 \mathrm{~A}-\mathrm{C}$ ), which ensures the RAA reaction specificity and provides alternative diagnostic conditions in practical applications.

The RAA-Cas12a-Tg system herein developed in this study is an ultrasensitive and robust platform for detection of pathogens. Some recent studies have verified that the signal amplification effect of Cas12a protein and the amplification procedure could make the 
fluorescence readout increase exponentially [48,49], and the sensitivity of our method is at femtomolar level (Figure 4A). Additionally, our method has the high specificity that mainly depends on the specific recognition of LbCas12a-crRNA targeting the single nucleotide mutations [50]. Compared with the conventional methods, our method is more sensitive and appropriate for point-of-care detection of environment samples without the need of sophisticated equipment. Therefore, this system can be used in laboratories with poor conditions or in the field.

While this new system has some advantages mentioned above, it still has some limitations. During the two separate processes, the potential cross contamination can be caused, thus the future research should mainly focus on the development of single-tube one-step detection which requires less time and more accuracy. Besides, the concentration of Cas12a used in this study were high, which increased the cost slightly. However, $200 \mathrm{nM}$ Cas12a protein still could work but the result was not as good as that of $800 \mathrm{nM}$ Cas12a. Nevertheless, this novel method is a new attempt to detect T. gondii in the environment, and it also can be applied to the detection of other pathogens.

\section{Conclusions}

RAA-Cas12a assay is a promising detection technology that is rapid, reliable, ultrasensitive and requiring no expensive instruments. Most importantly, this method can be performed at a low temperature environment, which makes it a practical on-site detection method to detect T. gondii in the field using a portable thermostatic heater and handheld fluorescence detector.

Author Contributions: X.-Q.Z., M.W. and X.-X.X. conceived and designed the study. Q.-N.M. performed the experiments, analyzed the data and drafted the manuscript. L.-B.Z., Z.-Q.L. and M.E. participated in the implementation of the study. X.-Q.Z., X.-X.X. and M.W. critically revised the manuscript. All authors have read and agreed to the published version of the manuscript.

Funding: This work was supported by the National Natural Science Foundation of China (Grant No. 32002306), the Fund for Shanxi "1331 Project" (Grant No. 20211331-13), the Agricultural Science and Technology Innovation Program (ASTIP) (Grant No. CAAS-ASTIP-2016-LVRI-03) and the Yunnan Expert Workstation (Grant No. 202005AF150041). The funders had no role in the design of the study; in the collection, analyses, or interpretation of data; in the writing of the manuscript, or in the decision to publish the results.

Institutional Review Board Statement: Not applicable.

Informed Consent Statement: Not applicable.

Data Availability Statement: The data that support the figures within this paper and other findings of this study are available from the corresponding authors upon reasonable request.

Acknowledgments: We thank Wei Cong of Marine College, Shandong University, China for providing 30 soil DNA samples.

Conflicts of Interest: The authors declare no conflict of interest.

\section{References}

1. Dubey, J.P. Toxoplasmosis of Animals and Humans, 2nd ed.; CRC Press: Boca Raton, FL, USA, 2010; pp. 1-313.

2. Zhou, P.; Chen, Z.; Li, H.-L.; Zheng, H.; He, S.; Lin, R.-Q.; Zhu, X.-Q. Toxoplasma gondii infection in humans in China. Parasit. Vectors 2011, 4, 165. [CrossRef]

3. Montoya, J.G.; Remington, J.S. Management of Toxoplasma gondii infection during pregnancy. Clin. Infect. Dis. 2008, 47, 554-566. [CrossRef]

4. Nissapatorn, V. Toxoplasma gondii and HIV: A never-ending story. Lancet HIV 2017, 4, e146-e147. [CrossRef]

5. Nayeri, T.; Sarvi, S.; Moosazadeh, M.; Daryani, A. Global prevalence of Toxoplasma gondii infection in the aborted fetuses and ruminants that had an abortion: A systematic review and meta-analysis. Vet. Parasitol. 2021, 290, 109370. [CrossRef]

6. Dawson, D. Foodborne protozoan parasites. Int. J. Food Microbiol. 2005, 103, 207-227. [CrossRef]

7. Simon, J.; Kurdzielewicz, S.; Jeanniot, E.; Dupuis, E.; Marnef, F.; Aubert, D.; Villena, I.; Poulle, M.-L. Spatial distribution of soil contaminated with Toxoplasma gondii oocysts in relation to the distribution and use of domestic cat defecation sites on dairy farms. Int. J. Parasitol. 2017, 47, 357-367. [CrossRef] 
8. Lass, A.; Ma, L.; Kontogeorgos, I.; Zhang, X.; Li, X.; Karanis, P. First molecular detection of Toxoplasma gondii in vegetable samples in China using qualitative, quantitative real-time PCR and multilocus genotyping. Sci. Rep. 2019, 9, 1-11.

9. Cong, W.; Zhang, N.Z.; Hu, R.S.; Zou, F.C.; Zou, Y.; Zhong, W.Y.; Wu, J.J.; Fallaize, C.J.; Zhu, X.Q.; Elsheikha, H.M. Prevalence, risk factors and genotype distribution of Toxoplasma gondii DNA in soil in China. Ecotoxicol. Environ. Saf. 2020, 189, 109999. [CrossRef] [PubMed]

10. Li, J.; Wang, Z.; Karim, R.; Zhang, L. Detection of human intestinal protozoan parasites in vegetables and fruits: A review. Parasit. Vectors 2020, 13, 380. [CrossRef] [PubMed]

11. Marques, C.; Sousa, S.; Castro, A.; Da Costa, J.M.C. Detection of Toxoplasma gondii oocysts in fresh vegetables and berry fruits. Parasit. Vectors 2020, 13, 180. [CrossRef] [PubMed]

12. Cong, W.; Li, M.-Y.; Zou, Y.; Ma, J.-Y.; Wang, B.; Jiang, Z.-Y.; Elsheikha, H.M. Prevalence, genotypes and risk factors for Toxoplasma gondii contamination in marine bivalve shellfish in offshore waters in eastern China. Ecotoxicol. Environ. Saf. 2021, 213, 112048. [CrossRef] [PubMed]

13. Deng, H.; Exel, K.E.; Swart, A.; Marinović, A.A.B.; Dam-Deisz, C.; van der Giessen, J.W.; Opsteegh, M. Digging into Toxoplasma gondii infections via soil: A quantitative microbial risk assessment approach. Sci. Total Environ. 2021, 755, 143232. [CrossRef] [PubMed]

14. Rousseau, A.; La, C.-S.; Dumètre, A.; Robertson, L.J.; Gargala, G.; Escotte-Binet, S.; Favennec, L.; Villena, I.; Gérard, C.; Aubert, D. Assessing viability and infectivity of foodborne and waterborne stages (cysts/oocysts) of Giardia duodenalis, Cryptosporidium spp., and Toxoplasma gondii: A review of methods. Parasite 2018, 25, 14. [CrossRef] [PubMed]

15. Liu, Q.; Wang, Z.-D.; Huang, S.-Y.; Zhu, X.-Q. Diagnosis of toxoplasmosis and typing of Toxoplasma gondii. Parasit. Vectors 2015, 8, 292. [CrossRef]

16. Elsheikha, H.M.; Marra, C.M.; Zhu, X.Q. Epidemiology, pathophysiology, diagnosis, and management of cerebral toxoplasmosis. Clin. Microbiol. Rev. 2021, 34, e00115-e00119.

17. Switaj, K.; Master, A.; Skrzypczak, M.; Zaborowski, P. Recent trends in molecular diagnostics for Toxoplasma gondii infections. Clin. Microbiol. Infect. 2005, 11, 170-176. [CrossRef] [PubMed]

18. Homan, W.; Vercammen, M.; De Braekeleer, J.; Verschueren, H. Identification of a 200- to 300-fold repetitive 529 bp DNA fragment in Toxoplasma gondii, and its use for diagnostic and quantitative PCR. Int. J. Parasitol. 2000, 30, 69-75. [CrossRef]

19. Valian, H.K.; Mirhendi, H.; Mohebali, M.; Shojaee, S.; Fallahi, S.; Jafari, R.; Kheirandish, F.; Mousavi, P. Comparison of the RE-529 sequence and B1 gene for Toxoplasma gondii detection in blood samples of the at-risk seropositive cases using uracil DNA glycosylase supplemented loop-mediated isothermal amplification (UDG-LAMP) assay. Microb. Pathog. 2020, 140, 103938. [CrossRef]

20. Calderaro, A.; Piccolo, G.; Gorrini, C.; Peruzzi, S.; Zerbini, L.; Bommezzadri, S.; Dettori, G.; Chezzi, C. Comparison between two real-time PCR assays and a nested-PCR for the detection of Toxoplasma gondii. Acta Bio-Medica Atenei Parm. 2006, 77, 75-80.

21. Jauregui, L.H.; Higgins, J.; Zarlenga, D.; Dubey, J.P.; Lunney, J.K. Development of a real-time PCR assay for detection of Toxoplasma gondii in pig and mouse tissues. J. Clin. Microbiol. 2001, 39, 2065-2071. [CrossRef]

22. Lin, Z.; Zhang, Y.; Zhang, H.; Zhou, Y.; Cao, J.; Zhou, J. Comparison of loop-mediated isothermal amplification (LAMP) and real-time PCR method targeting a 529-bp repeat element for diagnosis of toxoplasmosis. Vet. Parasitol. 2012, 185, 296-300. [CrossRef] [PubMed]

23. Wang, Y.; Wang, G.; Zhang, D.; Yin, H.; Wang, M. Detection of acute toxoplasmosis in pigs using loop-mediated isothermal amplification and quantitative PCR. Korean J. Parasitol. 2013, 51, 573-577. [CrossRef] [PubMed]

24. Sashital, D.G. Pathogen detection in the CRISPR-Cas era. Genome Med. 2018, 10, 32. [CrossRef]

25. Strich, J.R.; Chertow, D.S. CRISPR-Cas biology and its application to infectious diseases. J. Clin. Microbiol. 2019, 57, 01307-01318. [CrossRef] [PubMed]

26. Gootenberg, J.S.; Abudayyeh, O.O.; Kellner, M.J.; Joung, J.; Collins, J.J.; Zhang, F. Multiplexed and portable nucleic acid detection platform with Cas13, Cas12a, and Csm6. Science 2018, 360, 439-444. [CrossRef]

27. Gootenberg, J.S.; Abudayyeh, O.O.; Lee, J.W.; Essletzbichler, P.; Dy, A.J.; Joung, J.; Verdine, V.; Donghia, N.; Daringer, N.M.; Freije, C.A.; et al. Nucleic acid detection with CRISPR-Cas13a/C2c2. Science 2017, 356, 438-442. [CrossRef]

28. Harrington, L.B.; Burstein, D.; Chen, J.S.; Paez-Espino, D.; Ma, E.; Witte, I.P.; Cofsky, J.C.; Kyrpides, N.C.; Banfield, J.F.; Doudna, J.A. Programmed DNA destruction by miniature CRISPR-Cas14 enzymes. Science 2018, 362, 839-842. [CrossRef] [PubMed]

29. Bai, J.; Lin, H.; Li, H.; Zhou, Y.; Liu, J.; Zhong, G.; Wu, L.; Jiang, W.; Du, H.; Yang, J.; et al. Cas12a-based on-site and rapid nucleic acid detection of African swine fever. Front. Microbiol. 2019, 10, 2830. [CrossRef]

30. Chen, Y.; Mei, Y.; Zhao, X.; Jiang, X. Reagents-loaded, automated assay that integrates recombinase-aided amplification and Cas12a nucleic acid detection for a point-of-care test. Anal. Chem. 2020, 92, 14846-14852. [CrossRef]

31. Li, F.; Ye, Q.; Chen, M.; Zhou, B.; Zhang, J.; Pang, R.; Xue, L.; Wang, J.; Zeng, H.; Wu, S.; et al. An ultrasensitive CRISPR/Cas12a based electrochemical biosensor for Listeria monocytogenes detection. Biosens. Bioelectron. 2021, 179, 113073. [CrossRef]

32. Broughton, J.P.; Deng, X.; Yu, G.; Fasching, C.L.; Servellita, V.; Singh, J.; Miao, X.; Streithorst, J.A.; Granados, A.; Sotomayor-Gonzalez, A.; et al. CRISPR-Cas12-based detection of SARS-CoV-2. Nat. Biotechnol. 2020, 38, 870-874. [CrossRef]

33. He, Q.; Yu, D.; Bao, M.; Korensky, G.; Chen, J.; Shin, M.; Kim, J.; Park, M.; Qin, P.; Du, K. High-throughput and all-solution phase African swine fever virus (ASFV) detection using CRISPR-Cas12a and fluorescence based point-of-care system. Biosens. Bioelectron. 2020, 154, 112068. [CrossRef] 
34. Xiong, D.; Dai, W.; Gong, J.; Li, G.; Liu, N.; Wu, W.; Pan, J.; Chen, C.; Jiao, Y.; Deng, H.; et al. Rapid detection of SARS-CoV-2 with CRISPR-Cas12a. PLoS. Biol. 2020, 18, e3000978. [CrossRef]

35. Ai, J.-W.; Zhou, X.; Xu, T.; Yang, M.; Chen, Y.; He, G.; Pan, N.; Cai, Y.; Li, Y.; Wang, X.; et al. CRISPR-based rapid and ultra-sensitive diagnostic test for Mycobacterium tuberculosis. Emerg. Microbes Infect. 2019, 8, 1361-1369. [CrossRef] [PubMed]

36. Wang, Y.; Ke, Y.; Liu, W.; Sun, Y.; Ding, X. A one-pot toolbox based on Cas12a/crRNA enables rapid foodborne pathogen detection at attomolar level. ACS Sens. 2020, 5, 1427-1435. [CrossRef] [PubMed]

37. Zhou, J.; Yin, L.; Dong, Y.; Peng, L.; Liu, G.; Man, S.; Ma, L. CRISPR-Cas13a based bacterial detection platform: Sensing pathogen Staphylococcus aureus in food samples. Anal. Chim. Acta 2020, 1127, 225-233. [CrossRef]

38. Lee, R.A.; Puig, H.; Nguyen, P.Q.; Angenent-Mari, N.M.; Donghia, N.M.; McGee, J.P.; Dvorin, J.D.; Klapperich, C.M.; Pollock, N.R.; Collins, J.J. Ultrasensitive CRISPR-based diagnostic for field-applicable detection of Plasmodium species in symptomatic and asymptomatic malaria. Proc. Natl. Acad. Sci. USA 2020, 117, 25722-25731. [CrossRef]

39. Yu, F.; Zhang, K.; Wang, Y.; Li, D.; Cui, Z.; Huang, J.; Zhang, S.; Li, X.; Zhang, L. CRISPR/Cas12a-based on-site diagnostics of Cryptosporidium paroum IId-subtype-family from human and cattle fecal samples. Parasit. Vectors 2021, 14, 208. [CrossRef] [PubMed]

40. Lalitha, S.J.B.S. Primer Premier 5. Biotech Softw. Internet Rep. 2000, 1, 270-272. [CrossRef]

41. Zhang, L.; Sun, R.; Yang, M.; Peng, S.; Cheng, Y.; Chen, C. Conformational dynamics and cleavage sites of Cas12a are modulated by complementarity between crRNA and DNA. iScience 2019, 19, 492-503. [CrossRef]

42. Villena, I.; Aubert, D.; Gomis, P.; Ferté, H.; Inglard, J.-C.; Denis-Bisiaux, H.; Dondon, J.-M.; Pisano, E.; Ortis, N.; Pinon, J.-M. Evaluation of a strategy for Toxoplasma gondii oocyst detection in water. Appl. Environ. Microbiol. 2004, 70, 4035-4039. [CrossRef]

43. Swierzy, I.J.; Muhammad, M.; Kroll, J.; Abelmann, A.; Tenter, A.M.; Lüder, C.G. Toxoplasma gondii within skeletal muscle cells: A critical interplay for food-borne parasite transmission. Int. J. Parasitol. 2014, 44, 91-98. [CrossRef] [PubMed]

44. Harito, J.B.; Campbell, A.T.; Tysnes, K.R.; Dubey, J.P.; Robertson, L.J. Lectin-magnetic separation (LMS) for isolation of Toxoplasma gondii oocysts from concentrated water samples prior to detection by microscopy or qPCR. Water Res. 2017, 114, 228-236. [CrossRef]

45. Rousseau, A.; Escotte-Binet, S.; La Carbona, S.; Dumètre, A.; Chagneau, S.; Favennec, L.; Kubina, S.; Dubey, J.P.; Majou, D.; Bigot, A.; et al. Toxoplasma gondii oocyst infectivity assessed using a sporocyst-based cell culture assay combined with quantitative PCR for environmental applications. Appl. Environ. Microbiol. 2019, 85, e01189-19. [CrossRef] [PubMed]

46. Jian, D.; Wang, B.; Huang, H.; Meng, X.; Liu, C.; Xue, L.; Liu, F.; Wang, S. Sunlight based handheld smartphone spectrometer. Biosens. Bioelectron. 2019, 143, 111632. [CrossRef]

47. Mohanraju, P.; Mougiakos, I.; Albers, J.; Mabuchi, M.; Fuchs, R.T.; Curcuru, J.L.; van Kranenburg, R.; Robb, G.B.; van der Oost, J. Development of a Cas12a-based genome editing tool for moderate thermophiles. CRISPR J. 2021, 4, 82-91. [CrossRef]

48. Ding, X.; Yin, K.; Li, Z.; Lalla, R.V.; Ballesteros, E.; Sfeir, M.M.; Liu, C. Ultrasensitive and visual detection of SARS-CoV-2 using all-in-one dual CRISPR-Cas12a assay. Nat. Commun. 2020, 11, 4711. [CrossRef] [PubMed]

49. Shi, K.; Xie, S.; Tian, R.; Wang, S.; Lu, Q.; Gao, D.; Lei, C.; Zhu, H.; Nie, Z. A CRISPR-Cas autocatalysis-driven feedback amplification network for supersensitive DNA diagnostics. Sci. Adv. 2021, 7, eabc7802. [CrossRef]

50. Swarts, D.C. Making the cut(s): How Cas12a cleaves target and non-target DNA. Biochem. Soc. Trans. 2019, 47, 1499-1510. [CrossRef] 